\title{
Editorial: NAD Metabolism and Signaling in Plants
}

\author{
Alisdair Fernie ${ }^{1}$, Shin-nosuke Hashida ${ }^{2}$, Kazuya Yoshimura ${ }^{3}$, Bertrand Gakière ${ }^{4}$, \\ Zhonglin Mou ${ }^{5}$ and Pierre Pétriacq ${ }^{6 *}$ \\ 1 Department of Molecular Physiology, MPI of Molecular Plant Physiology, Potsdam-Golm, Germany, ${ }^{2}$ Environmental \\ Science Research Laboratory, Central Research Institute of Electric Power Industry (CRIEPI), Abiko-shi, Japan, ${ }^{3}$ Department \\ of Food and Nutritional Science, College of Bioscience and Biotechnology Chubu University, Kasugai, Japan, 4 Institute of \\ Plant Sciences Paris-Saclay (IPS2), UMR 9213/UMR1403, CNRS, INRAE, Université d'Evry, Université Paris-Diderot, \\ Université Paris-Sud, Sorbonne Paris-Cité, Saclay Plant Sciences, Orsay, France, ${ }^{5}$ Department of Microbiology and Cell \\ Science, University of Florida, Gainesville, FL, United States, ${ }^{6}$ Université de Bordeaux, INRAE, UMR BFP, Plateforme \\ Bordeaux Metabolome, Villenave d'Ornon, France
}

Keywords: plant, NAD signaling, pyridine nucleotide, NAD metabolism, fruit, cyanobacteria, light responce

Editorial on the Research Topic

\section{OPEN ACCESS}

Edited by:

Jean Rivoal,

Université de Montréal,

Canada

Reviewed by:

Abir U lgamberdiev,

Memorial University of Newfoundland,

Canada

Wayne Snedden,

Queen's University, Canada

*Correspondence:

Pierre Pétriacq

pierre.petriacq@inrae.fr

Specialty section:

This article was submitted to

Plant Physiology,

a section of the journal

Frontiers in Plant Science

Received: 10 December 2019

Accepted: 30 January 2020

Published: 26 February 2020

Citation:

Fernie A, Hashida S- $n$, Yoshimura K,

Gakière B, Mou Z and Pétriacq $P$

(2020) Editorial: NAD Metabolism and

Signaling in Plants.

Front. Plant Sci. 11:146.

doi: 10.3389/fp/s.2020.00146

\section{NAD Metabolism and Signaling in Plants}

Since its discovery more than a century ago and the conferral of several Nobel laureates for groundbreaking works concerning the molecule, nicotinamide adenine dinucleotide (NAD) is recognized as a fascinating metabolic cornerstone for all living organisms (Berger et al., 2004). This ubiquitous pyridine nucleotide (and its phosphorylated relative, NADP) is an energy cofactor and signalcarrying molecule exerting vital functions in plant metabolic pathways and regulatory processes (Hashida et al., 2009; Gakière et al., 2018b; Gakière et al., 2018a). Molecular studies on NAD involved transcript, protein, and metabolite profiling, and revealed a particularly complex signaling network that can impact not only the core biochemistry of the plant (e.g. photosynthesis, respiration, energy), but also responses to various stress, more importantly immune responses (Zhang and Mou, 2009; Pétriacq et al., 2012; Pétriacq et al., 2016; Alferez et al., 2018; Hashida et al., 2018). Remarkably, compelling evidence indicate that NAD can be released into the extracellular space, where it is sensed by cell surface receptors (Wang et al., 2017; Wang et al., 2019). However, the detailed mechanisms by which NAD acts as a regulator of plant physiology are still poorly understood. This Research Topic gathers some cardinal knowledge on the functions of NAD in plants.

Two papers in the issue focus on the key enzyme NAD kinase which phosphorylates $\mathrm{NAD}(\mathrm{H})$ forming $\mathrm{NADP}(\mathrm{H})$ (Gakière et al., 2018a). The first review by Ishikawa and Kawai-Yamada examined the roles of the enzyme in cyanobacteria-the evolutionary ancestor of land plant chloroplasts which has a far simpler level of compartmentation, in the oxidative pentose phosphate pathway, the glycolysis, and the pyridine nucleotide transhydrogenase proton translocation system. Survey of 72 cyanobacterial genomes revealed that 69 express a typical NAD kinase, raising the intriguing questions how typical NAD kinases contribute to $\mathrm{NAD}(\mathrm{P})(\mathrm{H})$ homeostasis in cyanobacteria and how this is regulated in the species containing the atypical NAD kinases. Also intriguing is the genetic identification of one of the latter as a factor linked to fast growth (Ungerer et al., 2018). However, a number of open questions remain, mainly why cyanobacteria need multiple NAD kinases and what the specific roles of the paralogs are. Further biochemical work alongside that on the membrane permeability of $\mathrm{NAD}(\mathrm{P}) \mathrm{H}$ will go a long way to addressing these questions. 
Secondly, Tai et al. reviewed the interplay of NAD kinases with calmodulin-mediated calcium signaling. They described the importance of the balance between $\mathrm{NADP}(\mathrm{H})$ and $\mathrm{NAD}(\mathrm{H})$ providing evidence suggesting that in response to allosteric regulation NAD kinase is a key regulator of the $\operatorname{NADP}(\mathrm{H})$ / $\mathrm{NAD}(\mathrm{H})$ ratio (Li et al., 2018). They then listed the evidence that calmodulin activates NAD kinase before performing a detailed analysis of wheat calmodulins, including predictions of gene and protein structures alongside subcellular localization and structure function studies. This provided a comprehensive overview of the putative regulatory control these proteins exhibit on NAD kinase (Tai et al.). Next, the review described the feedback regulation by $\mathrm{NAD}^{+}$derivatives, which is necessary for maintaining the dynamic balance of the cellular metabolites that ensure the normal operation of life activities.

Under sunlight, photosynthetic electron transfer chain is the primary source of NADPH as reducing power for assimilation of $\mathrm{CO}_{2}$ by the Calvin cycle. However, under stress conditions that weaken enzyme activity involved in the Calvin cycle, decline of $\mathrm{NADPH}$ usage and $\mathrm{NADP}^{+}$recycling occurs and photosynthetic electron flow can be overloaded resulting in generation of reactive oxygen species. Thus, the $\operatorname{NADP}(\mathrm{H})$ pool size and redox state are crucial for the chloroplastic redox balance. An important fact is that, at the beginning of photosynthesis, $\mathrm{NADP}^{+}$supply control is dominated by de novo $\mathrm{NADP}^{+}$ synthesis rather than recycling from the Calvin cycle. In addition, the activity of $\mathrm{NADP}^{+}$synthesis and the NADP pool size varies depending on the light conditions and the ferredoxinthioredoxin system, by which $\mathrm{NADP}^{+}$is reduced to NADPH. $\mathrm{NAD}^{+}$is exclusively produced in the cytosol, although $\mathrm{NADP}^{+}$is produced at on demand sites (cytosol, chloroplasts, and peroxisomes) by various isoforms of NAD kinase. Therefore, the regulatory mechanism of cytosolic $\mathrm{NAD}^{+}$supply is also involved in the chloroplastic $\mathrm{NADP}^{+}$supply control. Hashida and Kawai-Yamada summarized the regulatory mechanisms of $\mathrm{NADP}^{+}$production, focusing on the interactions, crosstalk, and co-regulation between chloroplasts and the cytoplasm at the level of $\mathrm{NAD}^{+}$metabolism and molecular transport.

NAD metabolism underlies a number of NAD-processing reactions. To start recycling NAD from nicotinate (NA), plants developed various strategies to overcome NA toxicity. Enzymatic conjugation of NA (e.g. glycosylation, methylation) has been discovered before and current metabolic analysis with much higher resolution and selectivity become available to examine NAD catabolites using mass spectrometry. Liu et al. identified two

\section{REFERENCES}

Alferez, F. M., Gerberich, K. M., Li, J.-L., Zhang, Y., Graham, J. H., and Mou, Z. (2018). Exogenous nicotinamide adenine dinucleotide induces resistance to Citrus Canker in Citrus. Front. Plant Sci. 9, 1472. doi: 10.3389/fpls.2018.01472

Berger, F., Ramírez-Hernández, M. H., and Ziegler, M. (2004). The new life of a centenarian: signalling functions of NAD(P). Trends Biochem. Sci. 29, 111-118. doi: 10.1016/j.tibs.2004.01.007

Decros, G., Baldet, P., Beauvoit, B., Stevens, R., Flandin, A., Colombié, S., et al. (2019). Get the balance right: ROS homeostasis and redox signalling in fruit. Front. Plant Sci. 10, 1091. doi: 10.3389/fpls.2019.01091 novel NA conjugates using a novel method, NA $\mathrm{N}$-pentoside and NA $N$-rhamnoside. Interestingly, they provided quantitative profiles of NAD-related metabolites in 24 plant tissues and biochemically characterized the responsible enzymes for conjugation with NA, being a key advance in our understanding of physiological significance of various NA conjugations.

NAD is also important for crops including fruits that constitute an important part of human diets, providing minerals, vitamins, fibers, and antioxidants that are essential for human health. Understanding of the biosynthetic production of these nutrients has attracted considerable attentions in recent years. Although it is well known that fruit development is associated with dramatic metabolic changes (Decros et al., 2019), how NAD metabolism and signaling are regulated during fruit development remains unclear. Decros et al. tackled this question via investigation of the changes in $\mathrm{NAD}(\mathrm{P})$ pools, transcriptome, and proteome profiles during nine growth stages of tomato fruit. The investigation revealed two critical links during tomato fruit development: a link between fine-tuned $\mathrm{NAD}(\mathrm{P})$ homeostasis and profound reprogramming of NAD (P) metabolism and a link between $\mathrm{NAD}(\mathrm{P})$-dependent enzymes and central metabolic pathways, particularly those involved in mitochondrial functions (Decros et al.). These results set up the stage for more in-depth investigations of the implication of NAD (P) in fruit development.

Hence, this Research Topic benefits the scientific community by providing new insights into the many aspects of NAD metabolism and signaling. Several open questions remain in order to understand fully the regulatory circuitry of NAD metabolism and signaling.

\section{AUTHOR CONTRIBUTIONS}

All authors wrote and/or reviewed the editorial.

\section{ACKNOWLEDGMENTS}

The editors wish to acknowledge the reviewers who handled one or more manuscripts for this Research Topic on NAD Signaling and Metabolism in Plants. Peer review of manuscripts is often underappreciated, yet crucial to the discipline and quality of scholarship published in this journal.

Gakière, B., Fernie, A. R., and Pétriacq, P. (2018a). More to NAD+ than meets the eye: a regulator of metabolic pools and gene expression in Arabidopsis. Free Radic. Biol. Med. 122, 85-96. doi: 10.1016/j.freeradbiomed.2018.01.003

Gakière, B., Hao, J., de Bont, L., Pétriacq, P., Nunes-Nesi, A., and Fernie, A. R. (2018b). NAD ${ }^{+}$Biosynthesis and Signaling in Plants. Crit. Rev. Plant Sci. 37, 1-49. doi: 10.1080/07352689.2018.1505591

Hashida, S.-N., Takahashi, H., and Uchimiya, H. (2009). The role of NAD biosynthesis in plant development and stress responses. Ann. Bot. 103, 819824. doi: 10.1093/aob/mcp019

Hashida, S.-N., Miyagi, A., Nishiyama, M., Yoshida, K., Hisabori, T., and KawaiYamada, M. (2018). Ferredoxin/thioredoxin system plays an important role in 
the chloroplastic NADP status of Arabidopsis. Plant J. Cell Mol. Biol. 95, 947960. doi: $10.1111 /$ tpj. 14000

Li, B.-B., Wang, X., Tai, L., Ma, T.-T., Shalmani, A., Liu, W.-T., Liu, W.-Q., and Chen, K.-M. (2018). NAD Kinases: metabolic targets controlling redox coenzymes and reducing power partitioning in plant stress and development. Front. Plant Sci. 9, 379. doi: 10.3389/fpls.2018.00379

Pétriacq, P., de Bont, L., Hager, J., Didierlaurent, L., Mauve, C., Guérard, F., et al. (2012). Inducible NAD overproduction in Arabidopsis alters metabolic pools and gene expression correlated with increased salicylate content and resistance to Pst-AvrRpm1. Plant J. 70, 650-665. doi: 10.1111/j.1365313X.2012.04920.x

Pétriacq, P., Ton, J., Patrit, O., Tcherkez, G., and Gakière, B. (2016). NAD acts as an integral regulator of multiple defense layers. Plant Physiol. 172, 1465-1479. doi: $10.1104 /$ pp. 16.00780

Ungerer, J., Wendt, K. E., Hendry, J. I., Maranas, C. D., and Pakrasi, H. B. (2018). Comparative genomics reveals the molecular determinants of rapid growth of the cyanobacterium Synechococcus elongatus UTEX 2973. Proc. Natl. Acad. Sci. 115, E11761-E11770. doi: 10.1073/pnas.1814912115

Wang, C., Zhou, M., Zhang, X., Yao, J., Zhang, Y., and Mou, Z. (2017). A lectin receptor kinase as a potential sensor for extracellular nicotinamide adenine dinucleotide in Arabidopsis thaliana. eLife 6, e25474. doi: 10.7554/ eLife. 25474

Wang, C., Huang, X., Li, Q., Zhang, Y., Li, J.-L., and Mou, Z. (2019). Extracellular pyridine nucleotides trigger plant systemic immunity through a lectin receptor kinase/BAK1 complex. Nat. Commun. 10, 4810. doi: 10.1038/s41467-019-12781-7

Zhang, X., and Mou, Z. (2009). Extracellular pyridine nucleotides induce PR gene expression and disease resistance in Arabidopsis. Plant J. 57, 302-312. doi: 10.1111/j.1365-313X.2008.03687.x

Conflict of Interest: The authors declare that the research was conducted in the absence of any commercial or financial relationships that could be construed as a potential conflict of interest.

Copyright $\odot 2020$ Fernie, Hashida, Yoshimura, Gakière, Mou and Pétriacq. This is an open-access article distributed under the terms of the Creative Commons Attribution License (CC BY). The use, distribution or reproduction in other forums is permitted, provided the original author(s) and the copyright owner(s) are credited and that the original publication in this journal is cited, in accordance with accepted academic practice. No use, distribution or reproduction is permitted which does not comply with these terms. 